\title{
Primary Malignant Peripheral Nerve Sheath Tumor of the Breast-A Case Report
}

\author{
Mushtaq Chalkoo, Shahnawaz Ahangar, Asim Rafiq Laharwal, \\ Aasim Mushtaq Patloo, Abbass Mohd, Shabir Ahmed Dar \\ Department of Surgery, Government Medical College, Srinagar, India \\ E-mail: Mushtaq_chalkoo@rediffmail.com; shanz101@gmail.com \\ Received February 19, 2011; revised April 3, 2011; accepted April 8, 2011
}

\begin{abstract}
Malignant peripheral nerve sheath tumor is a rare soft tissue sarcoma. Breast is an extremely rare location of this lesion and presentation as a breast lump in the absence of pain or previous benign neural tumor is even rarer. We report such a lesion in a 60-year-old female who presented with hard and painless breast lump for 2 years. Histopathology revealed a malignant spindle cell tumor of low grade potential. It was subsequently confirmed to be malignant peripheral nerve sheath tumor (MPNST) on the basis of immunopositivity for vimentin, neurone specific enolase and S-100.
\end{abstract}

Keywords: Breast Lump; Malignant Peripheral Nerve Sheath Tumor

\section{Introduction}

Breast tumors are predominantly of epithelial origin, and non-epithelial tumors arising from indigenous structures in the breast are rare [1]. Primary pure breast sarcoma is a rare disease and constitutes $0.2 \%-1.0 \%$ of all mammary malignancies [2]. The exact etiology is still unknown but these tumors frequently occur in patients suffering with neurofibromatosis Type I [NF 1]. The mean age at the time of diagnosis of MPNST is in the thirties, but the patients with NF 1 are about 10 yr younger than the patients without NF 1 [3]. MPNSTs are also known to have an association with previous irradiation. MPNSTs are known to arise within the field of irradiation, 9 to $36 \mathrm{yr}$ after radiation therapy administered for treating previous malignancies [4]. Immunohistochemical analysis proves to be helpful in indistinguishable cases. Though several cases of Malignant Peripheral Nerve Sheath Tumor (MPNST) of breast have been reported [5]. We report the first case with a one year follow up.

\section{Case Report}

A 60 years old female presented with a giant lump in her right breast for two years. The patient was concealing the lump by hiding it under her pajamas and tying a belt around it. It was only after the lump developed ulceration over its surface that the patient sought medical help. She had no previously significant medical or surgical history.
Her general physical examination was essentially normal. Breast examination revealed a giant lump involving whole of the right breast which had gravitated to the groin (Figure 1). Overlying skin was ulcerated along the inferio-medial aspect and the rest of the skin was hyper pigmented. It was firm in consistency and free from the underlying pectoral muscles. A solitary lymph node about $1 \times 1 \mathrm{~cm}$ was palpable in axilla with tenderness on palpation. Fine needle aspiration cytology [FNAC] of the lymph node revealed inflammatory changes. Patient was put on antibiotic treatment for a period of 5 days as per culture and sensitivity report of the wound swab from the

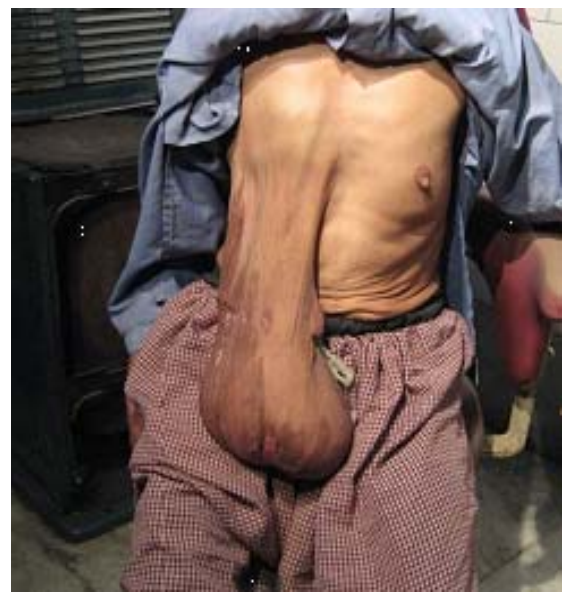

Figure 1. Preoperative photograph of the patient. 


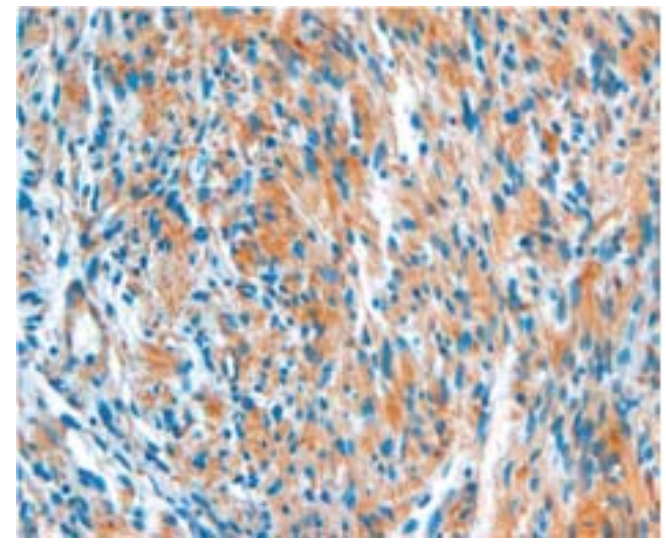

Figure 2. S-100 protein staining of the tumour tissue.

ulcer. Then simple mastectomy with excision of axillary node was contemplated. The excised surgical specimen consisted of a well-circumscribed, firm, solid mass measuring $26 \times 24 \times 18 \mathrm{~cm}$ covered with skin ellipse including nipple and areola. It weighed about $5 \mathrm{~kg}$. The cut section revealed grayish-white, nodular and myxoid mass with prominent fibrous strands. No necrosis, hemorrhage or cystic degeneration was visible. Histopathological examination revealed a malignant nerve sheath tumor of low grade potential, with focal infiltration by mixed inflammatory cell infiltrate. It was surrounded by fibrous tissue and spindle cells with numerous vascular channels. The mitotic count was 16 mitosis per 10 high-power fields. Immunohistochemical analysis showed positivity for neuron specific enolase (NSE) and vimentin and S-100 and expressed negativity for CD31, CD34, FACTOR VIII, PLAP, EMA, SMA and calponin (Figure 2). The excised lymph node revealed inflammatory changes only.

\section{Discussion}

Malignant peripheral nerve sheath tumour [MPNST] is a term that is used to describe tumours arising from the peripheral nerves or their sheaths and it has replaced the old terminology of schwanomas, malignant neurilemmomas and the like. Overall they represent about $10 \%$ of soft tissue sarcomas [6]. The incidence of MPNST in the general population is $0.001 \%$ however it can increase to $5 \%-42 \%$ in patients with neurofibromatosis type 1 . Peak incidence of these tumours is around 20 to 50 years [5]. Laskinet et al., reported pointed out to irradiation of peripheral nerves as one of the possible etiologies [7]. Such tumours develop after a long latent period (10 - 20 years) following irradiation and account for $11 \%$ of MPNST. Histologically, spindle-shaped cells with contours with curved cores and a slightly coloured cytoplasm are present. Cellular density and organization are variable and the palisade provision of the cores is found. On immunohistochemical staining, tumour cells show reactivity to neuron specific enolase (NSE) and Vimentin and S-100 [6]. The common sites of origin include the extremities and trunk usually sciatic nerve, brachial plexus and the sacral plexus. This tumour very rarely affects breast. To the best of our knowledge only 9 such cases have been reported with some of them being reported in association with neurofibromatosis-1 and post radiation [8-15]. In our case there was no clinical evidence of neurofibromatosis type 1 . Woo et al. noted similar presentation in their case [6]. The diagnosis of MPNST can only be done on the basis of immunohistochemistry otherwise it can be easily mistaken for malignant phylloides tumour, fibrosarcoma and leiomyosarcoma [5]. Owing to the rarity of the tumour there are no clear cut guidelines for its management. We performed a simple mastectomy in our patient. Catania et al. and Malas et al. have also recommended the same for the treatment of MPNST of breast [8,9]. Role of radiotherapy remains controversial. Yi et al. have tried adjuvant therapy in their case. However our patient did not undergo any radiation therapy [5]. One year into follow up the patient is doing well with no local recurrence or any distant metastasis. We have been following our patient with 6 monthly ultrasound abdomen and with a detailed history and physical examination every two months. There are no reports available in the literature on the median survival or the prognosis of MPNST of breast.

\section{Conclusions}

MPNST of breast is a very rare tumour. The optimum therapy needs to be charted out but we believe that it can be managed by simple mastectomy.

\section{References}

[1] B. Majmudar, "Neurilemoma Presenting as a Lump in the Breast,” Southern Medical Journal, Vol. 69, No. 4, April 1976, pp. 463-464. doi:10.1097/00007611-197604000-00025

[2] T. Cil, A. Altintas, S. Pasa and A. Isikdogan, "Primary Spindle Cell Sarcoma of the Breast,” Breast Care, Vol. 3, No. 3, 2008, pp. 197-199. doi:10.1159/000121469

[3] B. S. Ducatman, B. W. Scheithauer, D. G. Piepgras, H. M. Reiman and D. M. Ilstrup, "Malignant Peripheral Nerve Sheath Tumours: A Clinicopathologic Study of 120 Cases," Cancer, Vol. 57, No. 10, May 1986, pp. 2006-2021. doi:10.1002/1097-0142(19860515)57:10<2006::AID-CN CR2820571022>3.0.CO;2-6

[4] J. M. Baehring, R. A. Betensky and T. T. Batchelor, 
"Malignant Peripheral Nerve Sheath Tumour: The Clinical Spectrum and Outcome of Treatment," Neurology, Vol. 61, No. 5, September 2003, pp. 696-698.

[5] J. M. Yi, E. J. Moon, S. J. Oh, A. Lee , Y. J. Suh, J. M. Baek, S. H. Choi and S. S. Jung, "Malignant Peripheral Nerve Sheath Tumor of the Breast in a Patient without Neurofibromatosis: A Case Report," Journal of Breast Cancer, Vol. 12, No. 3, September 2009, pp. 223-226.

[6] O. H. Woo, H. S. Yong, J. B. Lee, A. Kim, B.H. Koo and E. Y. Kang, "A Giant Malignant Peripheral Nerve Sheath Tumour of the Breast: CT and Pathological Findings," British Journal of Radiology, Vol. 80, No. 950, 2007, pp. 44-47. doi:10.1259/bjr/30984808

[7] W. B. Laskin, T. A. Silverman and F. M. Enzinger, "Postradiation Soft Tissue Sarcomas. An Analysis of 53 Cases,” Cancer, Vol. 62, No. 11, December 1988, pp. 2330-2340. doi:10.1002/1097-0142(19881201)62:11<2330::AID-CN CR2820621113>3.0.CO;2-2

[8] S. Catania, E. Pacifico, S. Zurrida and F. Cusumano, "Malignant Schwannoma of the Breast," European Journal of Surgical Oncolgy, Vol. 18, 1992, pp. 80-81.

[9] S. Malas, H. E. Krawitz, R. K. Sur, R. R. Uijs, S. J. Nayler and C. V. Levin, "Von Recklinghausen's Disease Associated with a Primary Malignant Schwannoma of the Breast,” Journal of Surgical Oncolgy, Vol. 59, No. 4,
August 1995, pp. 273-275. doi:10.1002/jso.2930590415

[10] H. Medina-Franco, A. Gamboa-Dominguez and A. R. de la Medina, "Malignant Peripheral Nerve Sheath Tumor of the Breast,” Breast Journal, Vol. 9, No. 4, 2003, p. 332. doi:10.1046/j.1524-4741.2003.09420.x

[11] K. K. Dhingra, S. Mandal, S. Roy and N. Khurana, "Malignant Peripheral Nerve Sheath Tumor of the Breast: Case Report," World Journal of Surgical Oncology, Vol. 5, 2007, p. 142. doi:10.1186/1477-7819-5-142

[12] H. Hauser, A. Beham, P. Steindorfer, F. Schmidt and M. G. Smola, "Malignant Schwannoma of the Breast," Langenbecks Archives of surgery, Vol. 380, 1995, pp. 350-353.

[13] R. Berrada, A. Chahtane, A. Lakhdar, Z. Elhanchi, D. Ferhati, A. Baidada, A. Kharbach, F. Kettani and A. Chaoui, "Malignant Schwannoma of the Breast. A Case Report," Journal of Gynecology Obstetrics Biology and Reproduction, Vol. 27, 1998, pp. 441-444.

[14] I. Besznyaek, S. Dubecz and I. Peter, "Malignant Schwannoma of the Breast,” Orvosi Hetilap, Vol. 139, No. 18, January 1998, pp. 137-139.

[15] C. Thanapaisal, S. Koonmee and S. Siritunyaporn, "Malignant Peripheral Nerve Sheath Tumor of Breast in Patient without von Recklinghausen's Neurofibromatosis: A Case Report,” Journal of Medical Association of Thailand, Vol. 89, No. 3, March 2006, pp. 377-379. 